\title{
Modelagem matemática e simulação para previsão do fluxo de nêutrons em reatores nucleares
}

\section{Mathematical modeling and simulation for neutron flow prediction in nuclear reactors}

\author{
Carla Cristina Araújo Parreira ${ }^{1 *}$, Juan Canellas Bosch Neto $1^{2}$, Bruna Paloma Ribeiro 2 ${ }^{1}$, João \\ Victor Sales Castro $3^{1}$
}

\begin{abstract}
RESUMO
Nesse trabalho foi realizado um estudo e proposta para simulação da densidade de nêutrons em reatores nucleares. A viabilidade dos reatores nucleares depende de um sistema de segurança eficiente na operação de suas unidades de processamento nucleares. Grande parte da segurança dessas operações está relacionada com o fluxo de nêutrons nesses reatores, o que necessita de um balanço bem equilibrado entre os nêutrons emitidos e os nêutrons perdidos via absorção ou fuga de nêutrons, a fim de evitar grandes danos como superaquecimento e possíveis explosões. Neste trabalho, foram abordadas algumas técnicas para previsão desse fluxo de nêutrons dos reatores nucleares que podem permitir a aplicação de ferramentas de controle no sistema.
\end{abstract}

Palavras-chave: Fluxo de nêutrons; Simulação; Reator nuclear; Cinética pontual; Monte Carlo.

\begin{abstract}
In this work, a study and proposal for simulation of neutron density in nuclear reactors was carried out. The viability of nuclear reactors depends on an efficient safety system in the operation of their nuclear processing units. Much of the safety of these operations is related to the necessary neutron flux, which requires a well-balanced balance between the neutrons emitted and the neutrons lost via neutron absorption or leakage, in order to avoid major damage such as overheating and possible explosions. In this work, some techniques for predicting the neutron flux of nuclear reactors that can allow the application of control tools in the system were approached.
\end{abstract}

Keywords: Flow of neutrons; Simulation; Nuclear reactor; Punctual kinetics; Monte Carlo.

\footnotetext{
1. Universidade Federal de São João Del -carla-araujo07@hotmail.com

2. Universidade Federal de São João Del - jboschbr@ufsj.edu.br

3. Universidade Federal de São João Del-Rei - brunapalomaribeiro0016@ gmail.com

4. Universidade Federal de São João Del-Rei - jvsc55@gmail.com
} 


\section{INTRODUÇÃO}

A fim de reduzir uma parcela da emissão de gases de efeito estufa, cada vez mais a energia nuclear vem ganhando destaque em relação e energias provenientes de combustíveis fósseis. A energia nuclear é uma energia que pode ser considerada limpa, quando comparada com outras formas de energia provenientes de combustíveis fósseis. Esse tipo de energia tem apresentado um papel crucial para suprir demandas energéticas globais e principalmente evitar danos relacionados ao clima causados por efeito da eliminação de gases de efeito estufa, por exemplo o dióxido de carbono liberado durante a queima de combustíveis fósseis.

Um dos grandes desafios atuais para a geração de energia nuclear é o controle e segurança das usinas nucleares. Após os graves acidentes nucleares, como Three Mile Island em 1979 e Chernobyl em 1986, as questões de segurança dos reatores nucleares ganharam ainda mais destaque nas pesquisas. $\mathrm{O}$ acidente de Three Mile Island ocorreu devido à perda de líquido refrigerante que levou ao derretimento parcial do reator, devido ao aumento exponencial do fluxo de nêutrons sem um agente moderador que pudesse controlar esse fluxo e o consequente aumento da temperatura no reator. $\mathrm{O}$ acidente de Chernobyl ocorreu durante um teste de segurança que simulava uma falta de energia, o que resultou em condições de reações descontroladas. Diante desse descontrole do sistema, o fluxo de nêutrons não conseguiu ser moderado pelo líquido de arrefecimento e aumentou a temperatura do reator, fazendo com que o vapor superaquecido elevasse consideravelmente a pressão do sistema reacional, o que conduziu um rompimento do vaso de pressão através de uma explosão. Diante do exposto, fica clara a necessidade de um bom controle do fluxo de nêutrons que são os responsáveis pelas fissões nos reatores e consequente aquecimento, para evitar situações que possam levar a acidentes como esses. Diante desses fatos foi necessário que houvesse cada vez mais, tecnologias que proporcionasse um equilíbrio satisfatório entre segurança e economia nas usinas nucleares.(DONG et al., 2017)

Para a utilização de reatores nucleares de forma segura, minimizando a chance de superaquecimento e possíveis acidentes, e que seja também economicamente viável, é necessário que exista estudos a respeito do projeto da usina nuclear e do próprio reator, relacionado ao tipo de reator (sendo o mais utilizado atualmente o reator de água pressurizada) e à sua estrutura, por exemplo, em termos dos circuitos de refrigeração 
bem planejados. Também é essencial uma análise neutrônica bem elaborada, visto que os nêutrons são os essenciais nesse processo (SHAUKAT; RYU; SHIM, 2017).

De acordo com Shaukat, et al (2017), existem vários parâmetros que podem alterar a reatividade de um reator nuclear, como a temperatura de operação e o consumo do combustível. Sendo assim, é fundamental que esses parâmetros sejam mantidos constantes, a fim de obter maior segurança na operação do reator. A maior parte dos dados nucleares necessários para análise de reatores (Tabela 1) tem sido medida e avaliada por metodologia computacional, como a técnica das diferenças finitas e métodos estatísticos como a Técnica de Monte Carlo ou calculada por meio de equações cinéticas que calculam o fluxo de nêutrons como a equação 1 que corresponde à equação diferencial de (Z. MESQUITA; C. D. LADEIRA; A. P. PALMA, 2016).

$$
\frac{d N(t)}{d t}=\frac{\rho(t)-\beta}{\Lambda} N(t)+\sum_{i=1}^{6} \lambda i C i(t)
$$

Em que:

$\mathrm{dN}(\mathrm{t}) / \mathrm{dt}=$ Fluxo de nêutrons

$\rho(t)=$ reatividade dos nêutrons atrasados

$\beta=$ grupo precursores nêutrons atrasados

$\Lambda=$ tempo dos nêutrons térmicos

$\lambda=$ constante de decaimento do tempo de meia vida

$\mathrm{Ci}=$ concentração do grupo de nêutrons retardados

Como exemplo, alguns desses dados nucleares, são relacionados aos produtos de fissão que emitem nêutrons atrasados. Na tabela abaixo foram reunidos os dados de 6 grupos de nêutrons atrasados de acordo com sua meia-vida. 
Table 1- Parâmetros nucleares dos precursores de nêutrons atrasados para alguns núcleos

\begin{tabular}{c|c|c}
\hline Grupo & $\left.\boldsymbol{\lambda i \boldsymbol { i }} \mathbf{s}^{\mathbf{- 1}}\right)$ & Fração $\boldsymbol{\beta i}$ \\
\hline $\mathbf{U}_{\mathbf{2 3 5}}$ & & 0,000022 \\
2 & 0,0124 & 0,00142 \\
3 & 0,0305 & 0,00127 \\
4 & 0,111 & 0,00257 \\
5 & 0,310 & 0,00075 \\
6 & 1,14 & 0,00027 \\
\hline Pu239 & 3,01 & \\
\hline 1 & & 0,000076 \\
2 & 0,0129 & 0,000560 \\
3 & 0,0311 & 0,000432 \\
4 & 0,134 & 0,000656 \\
5 & 0,331 & 0,000206 \\
6 & 1,26 & 0,000070 \\
\hline $\mathbf{U}_{\mathbf{2 3 3}}$ & 3,21 & \\
\hline 1 & & 0,00023 \\
2 & 0,0126 & 0,00081 \\
3 & 0,0337 & 0,00068 \\
4 & 0,139 & 0,00075 \\
5 & 0,305 & 0,00014 \\
\hline
\end{tabular}

Fonte: Z. MESQUITA; C. D. LADEIRA; A. P. PALMA (2016)

Cada vez mais, é possível, por meio das modelagens e simulações predizer com acurácia o comportamento físico dos reatores nucleares, apesar de não ser de grande facilidade.

Nesse artigo será abordada a modelagem dinâmica e a simulação de reatores nucleares aplicada ao fluxo de nêutrons e à potência do reator, destacando que esse rastreamento de nêutrons para análise da potência do reator requer métodos de resolução eficientes, como as técnicas apresentadas, para controle de nêutrons atrasados e nêutrons rápidos. É grande o interesse nos resultados de qualidade das equações provenientes da cinética, assim como os esforços para quantificar e reduzir incertezas provenientes de cálculos computacionais teóricos. Essas incertezas estão relacionadas com a quantidade de simulações realizadas, sendo quanto mais simulações menores as incertezas relacionadas ao processo. As duas técnicas são apresentadas a seguir: 


\section{DETERMINAÇÃO DO FLUXO DE NÊUTRONS POR MEIO DA CINÉTICA PONTUAL}

No estudo dos problemas de cinética, pode-se em geral admitir que o fluxo neutrônico varia em amplitude sem mudar de distribuição espacial. Esta hipótese permite limitar o estudo da cinética ao único fator temporal, ou, em outros termos, como se o reator estivesse reduzido a um ponto, e por isso essa técnica é comumente conhecida por cinética pontual. Esse método é utilizado para cálculos da potência do reator provenientes dos dados do fluxo de nêutrons e são gerados sistemas numéricos com inúmeras equações diferenciais a serem solucionadas.

Voese, et al (2018) relatam que o modelo de cinética pontual tem um papel relevante para a compreensão da cinética dos reatores na medida em que pode ser utilizado, quando devidamente resolvido, para uma previsão de tempo quase real da potência do reator, o que permite um controle de tempo e possível intervenção na operação, a fim de evitar a ocorrência de acidentes.

Segundo Voese et al (2018), foi citado que Duderstadt e Hamilton utilizaram para determinação do fluxo de neutrons, o Sistema de equações diferenciais que é composto também por equações que descrevem a concentração de nêutrons atrasados que são provenientes do decaimento de produtos de fissão e que interferem na densidade total dos neutrons no Reator. Considerando que existem 6 grupos de precursores de neutrons atrasados, chega-se normalmente a um Sistema com 6 equações diferenciais, partindo da Equação 2, onde ocorrerá variações de acordo com o índice i que representará cada grupo de neutrons atrasados de 1 a 6. (Z. MESQUITA; C. D. LADEIRA; A. P. PALMA, 2016).

$$
\frac{d \mathrm{Ci}}{\mathrm{dt}}=\frac{\beta i}{\Lambda} \mathrm{N}(\mathrm{t})-\lambda i \partial \mathrm{Ci}
$$

2

Em que:

$\mathrm{dCi} / \mathrm{dt}=$ Variação da concentração

$\beta=$ fração de precursores nêutrons atrasados

$\Lambda=$ tempo dos nêutrons térmicos 
$\mathrm{N}=$ fluxo de nêutrons

$\lambda=$ constante de decaimento do tempo de meia vida dos grupos

$\mathrm{Ci}=$ concentração do grupo de nêutrons atrasados

Também é possível realizar o cálculo da potência do reator:

$\frac{d \partial \mathrm{P}}{\mathrm{dt}}=\frac{\rho-\beta}{\Lambda} \partial \mathrm{P}+\sum_{1}^{6} \lambda i \partial \mathrm{Ci}$

Equação 3

Em que:

$\mathrm{d} \partial \mathrm{P} / \mathrm{dt}=$ Variação da Potência do reator

$\rho(t)=$ reatividade dos nêutrons atrasados

$\beta=$ fração de precursores nêutrons atrasados

$\Lambda$ = tempo dos nêutrons térmicos

$\mathrm{P}$ é a potência do reator

$\lambda=$ constante de decaimento do tempo de meia vida

$\mathrm{Ci}$ é a concentração do grupo de nêutrons retardados

\section{DETERMINAÇÃO DO FLUXO DE NÊUTRONS PELO MÉTODO DE MONTE} CARLO

Ao contrário do que se faz na Cinética pontual, que parte do modelo matemático do sistema através da discretização de equações diferenciais, o método de Monte Carlo utiliza técnicas de amostragem para se encontrar a solução do problema e tem sido cada vez mais utilizado com o avanço dos recursos computacionais. Muitas vezes o processo pode ser simulado sem a necessidade de descrever as equações matemáticas que o representam, sendo necessário que esse processo seja descrito por valores estimados de acordo com a probabilidade de ocorrência dos mesmos. Assim, uma vez que estes valores seja suposto, a simulação de Monte Carlo é realizada através de sua amostragem aleatória, e então o resultado desejado é obtido através da estimativa do valor médio das grandezas observadas durante um determinado número de simulações de acordo com tentativas. A desvantagem do método, de acordo com Alaskary e El-Beltagy (2020) é que ele necessita de muitas simulações e, portanto, consome muito tempo 
computacional, principalmente quando é necessário obter resultados com grande acurácia, como no caso de Reatores Nucleares. Isto porque para diminuir, em parte, a incerteza estatística obtida como resultado do método de Monte Carlo é necessário aumentar em muitas vezes a quantidade de simulações, aumentando em muito o tempo computacional, porque para aumentar em dez vezes a acurácia, devem-se fazer cem vezes mais simulações. O código MCNP, desenvolvido e mantido pelo Laboratório Nacional de Los Alamos, EUA (Los Alamos National Laboratory), é o código reconhecido internacionalmente para análise de transporte de nêutrons pelo método de Monte Carlo. (MOLNAR; TOLNAI; LEGRADY, 2019).

Böröczki, et al. (2020) afirmam que os códigos cinéticos do reator, como o MCNP, são cruciais na avaliação de segurança e que a validação dos solucionadores cinéticos de alta resolução espacial e temporal é problemático, pois as medições raramente envolvem resolução espacial e temporal fina detalhada. Em seu trabalho tentaram comparar os resultados do código da técnica Monte Carlo dependente do tempo com outros resultados da literatura e apesar de terem obtido êxito na obtenção dos dados, concluíram que houve uma grande incerteza nesses resultados comparados a dados reais monitorados. Estas discrepâncias podem ser atribuídas a deficiências nos modelos e muitas aproximações nas soluções numéricas.

Shaukat, et al. (2017) analisaram um novo método de simulação em estado estacionário baseado no rastreamento de nêutrons dependente do tempo. Durante este processo, fontes de nêutrons imediatos e precursores de nêutrons atrasados para a simulação de transiente foram amostrados. O método foi implementado conforme técnica de Monte Carlo e aplicado para problemas de cinética de núcleo bidimensional no transporte de nêutrons dependente do tempo.

\section{RESULTADOS E DISCUSSÃO}

Voese, et al. (2018) utilizaram o método da cinética pontual considerando dois casos para a reatividade, sendo uma reatividade linear e outra senoidal e chegaram à conclusão de que a técnica obteve uma boa concordância em praticamente todos os aspectos analisados quando comparados com o mesmo método resolvido por um programa computacional diferente descrito na literatura. Então com esse trabalho foi possível apresentar uma solução analítica para as equações de cinética pontual com 6 
grupos de precursores de nêutrons atrasados, utilizando o método da aproximação polinomial (MAP) via série de potências. Foi considerado que a solução em série de potências são facilmente manipuláveis, tanto analítica quanto numericamente, o que torna o método de fácil implementação. Além disso, o método mostrou-se eficiente e preciso para resolver as equações de cinética pontual. Porém os únicos cálculos realizados foram em relação à densidade de nêutrons, não realizando os cálculos dos nêutrons antes e após a moderação dos mesmos e também não mostrando os cálculos da potência do reator.

Polo-Labarrios et al. (2020) realizaram os cálculos da densidade de nêutrons quando a potência do reator nuclear é aumentada usando a equação da cinética pontual de nêutron fracionário considerando um único grupo de precursor de nêutron atrasado. Chegaram à conclusão que para uma grande escala de tempo, os resultados mostraram que a equação clássica da cinética pontual de nêutron maximizou a real densidade de nêutrons. Também não realizaram os cálculos relacionados à potência do reator e consideraram apenas um grupo de geradores de nêutrons atrasados o que com certeza implicará numa alteração dos valores quando comparados à realidade, afinal existem seis grupos de precursores de nêutrons atrasados que são responsáveis por uma pequena, mas significativa, parcela da densidade de nêutrons.

Böröczki, et al. (2020) utilizaram a técnica de Monte Carlo e os resultados mostraram uma boa correspondência com outros resultados, embora a fidelidade da comparação pudesse ser melhorada reduzindo a incerteza estatística dos resultados fornecidos, o que já foi citado como a maior desvantagem desse método. Também não foi citada nenhuma análise referente ao grupo de precursores de nêutrons atrasados.

Macconnachie e Novog (2021) afirmaram que os resultados do cálculo pela técnica de Monte Carlo mostraram boa concordância com os resultados de um código de análise de transporte determinístico encontrado na literatura, conforme relatado por Shaukat, et al. (2017), porém também não demonstraram os cálculos relacionados à potência do reator, além de ter sido necessário um enorme trabalho computacional.

O conhecimento da densidade de nêutrons em um reator, que pode responder a inúmeras perturbações, é moderada para que se mantenha o reator dentro dos padrões de segurança já mencionados. Infelizmente, essa informação é difícil de ser obtida e para estudar o comportamento de reatores nucleares é necessário resolver o modelo de 
difusão de nêutrons que é um sistema de equações diferenciais parciais acopladas e portanto resoluções complexas (ABOANBER; NAHLA; ALJAWAZNEH, 2021).

Aboanber e colaboradores (2021) acreditam que o modelo fracionário das equações de cinética de ponto pode ser uma boa representação para a densidade de nêutrons nos reatores nucleares. Esse modelo, também citado por Voese (2018) é o método utilizado para cálculos da potência do reator provenientes dos dados do fluxo de nêutrons e são gerados sistemas numéricos com inúmeras equações diferenciais a serem solucionadas.

Em seu trabalho, as equações de cinética de ponto fracionário de dois grupos de energia com multi-grupo de precursores de nêutrons atrasados são formuladas na forma de matriz e duas técnicas são propostas para resolver este sistema de equação diferencial analiticamente, que se baseia na transformação de Laplace e nos autovalores e os autovetores correspondentes da matriz de coeficientes. O procedimento analítico para o modelo fracionário proposto na dinâmica de reatores nucleares é descrito e investigado para diferentes tipos de reatividade variável no tempo. O desempenho dos métodos desenvolvidos foi testado para densidade de nêutrons rápidos e térmicos no caso da fonte de nêutrons dependente do tempo.

A figura 1 (ABOANBER, et al., 2021) abaixo, retrata o comportamento do fluxo de nêutrons dentro do reator, mostrando um crescimento exponencial dessa densidade de nêutrons., mostrando a necessidade desse fluxo ser moderado.

Figura 1 - Densidade de nêutrons rápidos
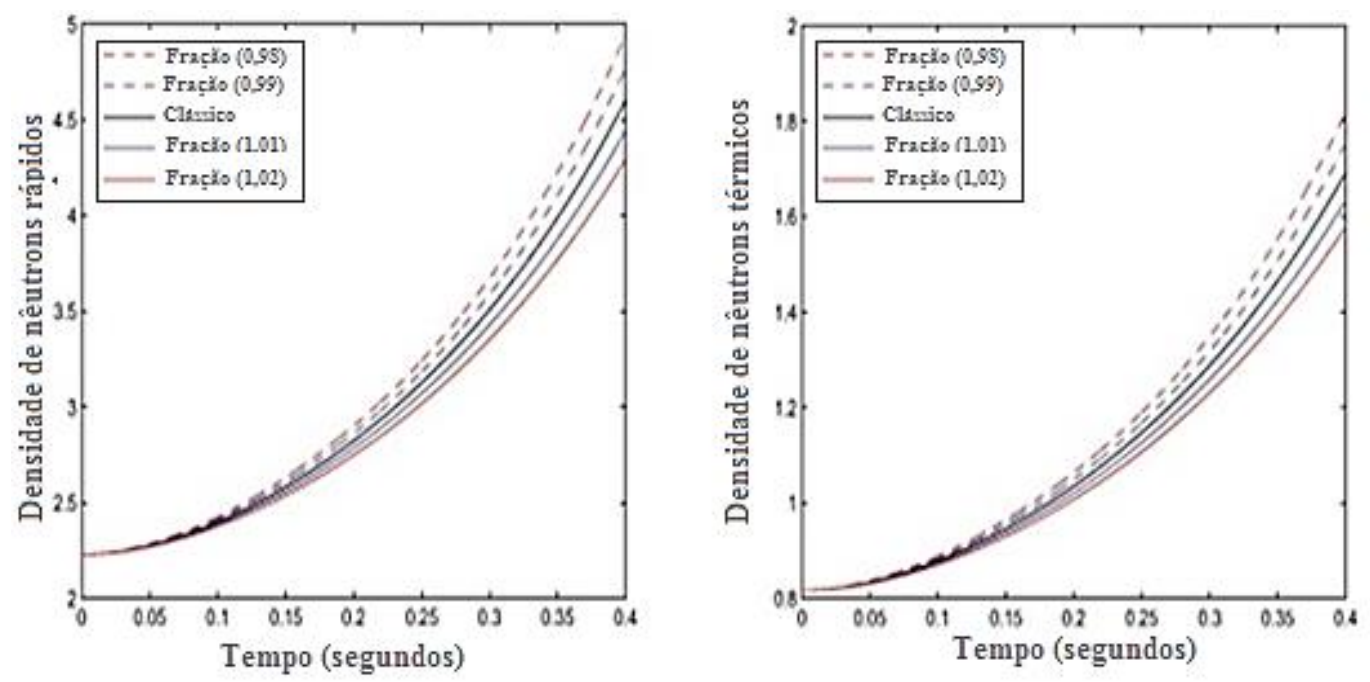

Fonte: ABOANBER, et al. (2021) 
Alaskary e El-Beltagy (2020) testaram uma técnica híbrida que permite derivar um sistema de equações que pode ser resolvido para obter o comportamento do fluxo de nêutrons em dois tipos de reatores, conforme demonstrado na Figura 2 abaixo, dos autores (ALASKARY; EL-BELTAGY, 2020), que demonstra o mesmo comportamento de crescimento, porém não de forma exponencial como demonstrado por ABOANBER, et al., 2021.

Figure 2 - Fluxo de nêutrons no reator

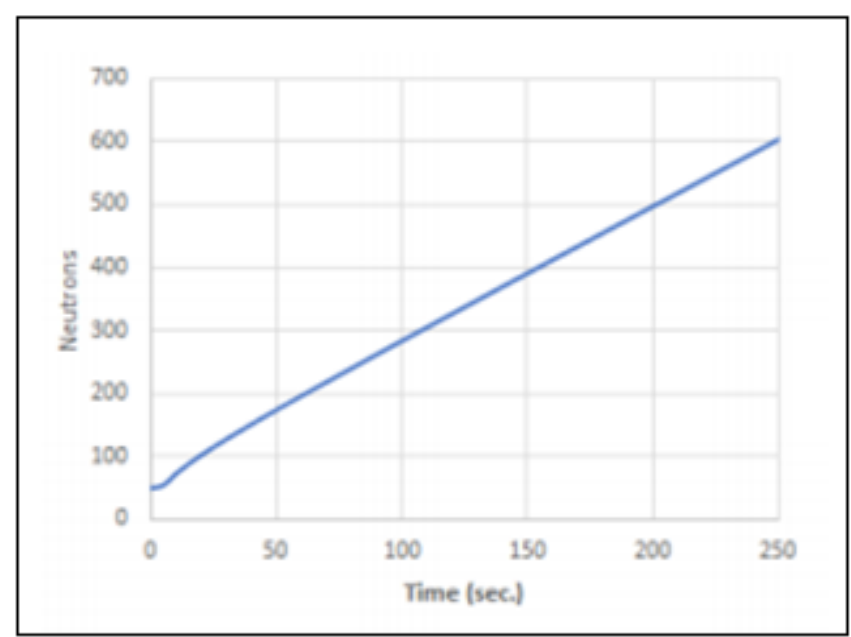

Fonte: ALASKARY; EL-BELTAGY (2020)

Validar simulações computacionais de alta resolução espacial e temporal é algo complexo, pois as medições raramente envolvem resolução espacial detalhada e resolução temporal. Boroczki e colaboradores (2020) simularam um cenário hipotético envolvendo uma inserção instantânea de moderadores de nêutrons a fim de comparar os fluxos temporalmente. Os resultados das simulações demonstraram uma boa correspondência com outros resultados realizados a partir dos códigos de cinética de reator Monte Carlo. A figura 3 explicita o que ocorre com a potência do reator (diretamente relacionada ao fluxo de nêutrons) quando simulam a inserção e a retirada parcial dos absorvedores de nêutrons. Esse comportamento deixa claro que sem os absorvedores a potência teria o mesmo comportamento do fluxo de nêutrons sem moderação que seria de um crescimento exponencial, demonstrando a tamanha importância desse processo de controle do aumento do fluxo e da potência do reator para evitar superaquecimento e possíveis explosões. 
Figura 3 - Potência do reator com inserção e retirada de absorvedores

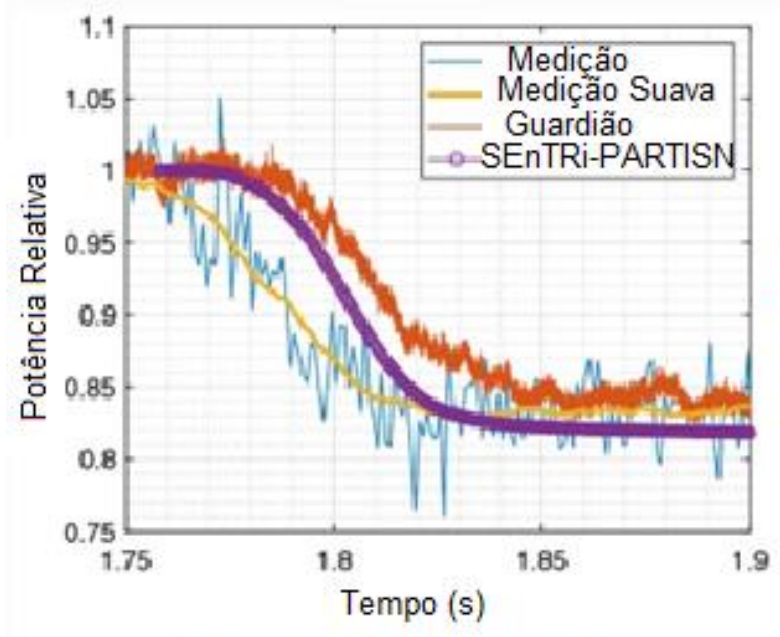

a) Inserção do absorvedor

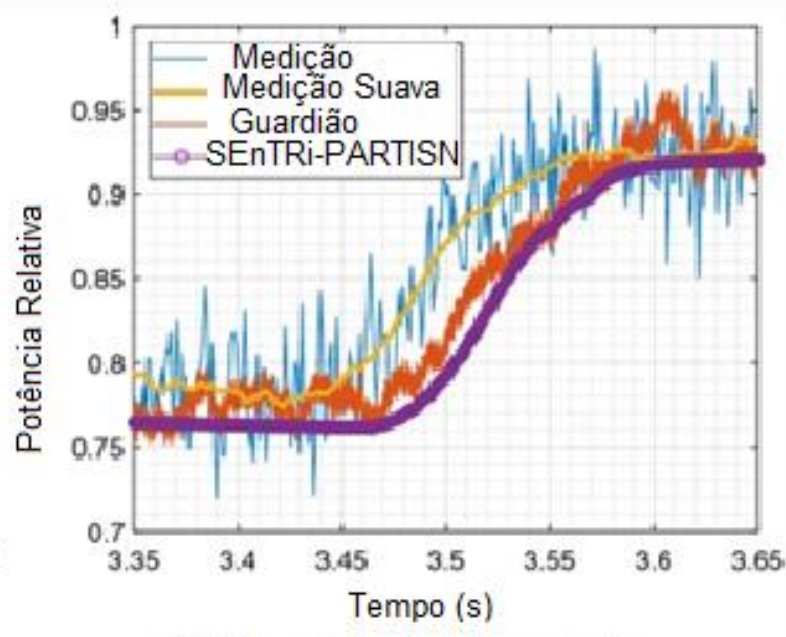

b) Remoção do absorvedor

Fonte: BÖRÖCZKI et al. (2020)

Pode-se analisar por essa Figura que com a inserção de uma fonte absorvedora de nêutrons, ocorre uma moderação do fluxo de nêutrons e consequentemente uma diminuição da potência do Reator e com a retirada desses absorvedores, volta a ocorrer um aumento da população neutrônica e da potência.

Com essa revisão, percebe-se que os métodos apresentados da cinética pontual e técnicas computacionais são muito utilizados para previsão de fluxo de fluxo de nêutrons, porém apresentam desvantagens e carências citadas durante o texto, com isso nota-se a necessidade de elaboração de outras técnicas com essa mesma finalidade, diante da importância dessas simulações para compreensão e controle dos processos nucleares.

\section{CONCLUSÃO}

Tanto os métodos que utilizam a cinética para realizar os cálculos do fluxo de nêutrons como os métodos que se baseiam na fluidodinâmica computacional são métodos bastante utilizados, porém extremamente difíceis de ser resolvidos. É necessário que se tenha um método mais simples e certeiro para essa análise e que também seja viável para descrever o fluxo de nêutrons moderados, após a inserção de moderados no reator. Outra carência é em relação aos cálculos considerando os seis grupos de precursores de nêutrons atrasados que interferem na densidade neutrônica. E 
por fim é viável que nas modelagens contemplem o cálculo final relacionando o fluxo de nêutrons com a potência do reator. Em suma, a elaboração de novas ferramentas matemáticas que possibilitem o controle e a avaliação de segurança nos processos de fissão nuclear é algo de extrema relevância e importância e ainda hoje se faz necessário uma técnica que consiga abordar todos os quesitos supracitados.

\section{REFERÊNCIAS}

ABOANBER, A. E.; NAHLA, A. A.; ALJAWAZNEH, S. M. Fractional two energy groups matrix representation for nuclear reactor dynamics with an external source. Annals of Nuclear Energy, v. 153, 1 Apr. 2021.

ALASKARY, S.; EL-BELTAGY, M. Uncertainty quantification spectral technique for the stochastic point reactor with random parameters. Energies, v. 13, n. 6, 2 Mar. 2020.

BÖRÖCZKI, Z. I. et al. Simulation of a research reactor reactivity transient with deterministic and GPU-assisted Monte Carlo reactor kinetics codes. European Physical Journal Plus, v. 135, n. 3, 2020.

DONG, Z. et al. Dynamic modeling and control characteristics of the two-modular HTR-PM nuclear plant. Science and Technology of Nuclear Installations, v. 2017, 2017.

MACCONNACHIE, E. L.; NOVOG, D. R. Measurement, simulation and uncertainty quantification of the neutron flux at the McMaster Nuclear Reactor. Annals of Nuclear Energy, v. 151, 2021.

MOLNAR, B.; TOLNAI, G.; LEGRADY, D. A GPU-based direct Monte Carlo simulation of time dependence in nuclear reactors. Annals of Nuclear Energy, v. 132, 2019.

POLO-LABARRIOS, M. A. et al. Novel numerical solution to the fractional neutron point kinetic equation in nuclear reactor dynamics. Annals of Nuclear Energy, v. 137, 2020.

SHAUKAT, N.; RYU, M.; SHIM, H. J. Dynamic Monte Carlo transient analysis for the Organization for Economic Co-operation and Development Nuclear Energy Agency (OECD/NEA) C5G7-TD benchmark. Nuclear Engineering and Technology, v. 49, n. 5, p. 920-927, 1 Aug. 2017.

VOESE, J.; TUMELERO, F.; PETERSEN, C. Solução das equações de cinética pontual de nêutrons via aproximação polinomial com o uso da continuação analítica, 32. n. 1, 2018. 
Z. MESQUITA, A.; C. D. LADEIRA, L.; A. P. PALMA, D. An Innovative Method for Online Power Monitoring in Nuclear Reactors. Recent Patents on Signal Processing, v. 5, n. 1, p. 61-67, 2016.

Recebido em: 15/09/2021

Aprovado em: 10/10/2021

Publicado em: 20/10/2021 